\title{
Por una psiquiatría experimental y de laboratorio: la formación de una comunidad alemano-brasileña de la medicina mental (1900-1914)*
}

\section{Towards the Experimental and Laboratorial Psychiatry: The Constitution of a German-Brazilian Community in Mental Medicine (1900-1914)}

Recibido: enero 21 de 2014 | Revisado: julio 26 de 2014 | Aceptado: julio 27 de 2014

\author{
Pedro Felipe Neves de MuÑoz ** \\ Casa de Oswaldo Cruz, Fundação Oswaldo Cruz, \\ Rio de Janeiro, Brasil
}

doi:10.11144/Javeriana.upsy13-5.ppel

Para citar este artículo: Muñoz, P. F. N., de (2014). Por una psiquiatría experimental y de laboratorio: la formación de una comunidad alemano-brasileña de la medicina mental (1900-1914). Universitas Psychologica, 13(5), 1967-1981. http://dx.doi.org/10.11144/ Javeriana.upsy13-5.ppel

Agradezco a CAPES y al DAAD por finaciar mis estudios y mi investigación en Brasil y en Alemania. Este artículo es una discusión introductoria a mi tesis de doctorado, cuya investigación estudia las comunidades psiquiátricas de Brasil y Alemania en el período de 1919 a 1942 y se completará en mayo de 2015.

* Doctorando en Historia de las Ciencias y de la Salud (Casa de Oswaldo Cruz/Fundação Oswaldo Cruz) y doctorando visitante CAPES/DAAD, en el Lateinamerika-Institut (LAI) de la Freien Universität Berlin (FU). Dirección postal: Rua Jorge Rudge 78, casa 07/201 - Vila Isabel, Rio de Janeiro -RJ, Brasil.E-mail: pedrodemunoz@hotmail.com

\section{RES U M E N}

El artículo analiza la formación de una comunidad psiquiátrica transnacional, donde médicos brasileños y alemanes realizaron un intenso intercambio científico, especialmente después de la Primera Guerra Mundial. En Alemania, Emil Kraepelin (1856-1926) dio nuevas directrices para la investigación psiquiátrica y estableció un modelo de producción del conocimiento psicológico y neuropsiquiátrico, a través de diversas especialidades científicas. En Brasil, los médicos Juliano Moreira (1873-1933) y Ulysses Vianna (1880-1935) fueron responsables de la apropiación del programa kraepeliano y de la circulación de médicos, conocimientos y modelos institucionales, entre los dos países. En el campo de la medicina mental, se constata el desarrollo de una nueva forma de producción de conocimiento científico, con énfasis en laboratorio, y de acercamiento con el paradigma biológico y experimental.

Palabras clave

historia de la psiquiatría; Kraepelin; Brasil-Alemania.

\section{A B S T R A C T}

This article analyzes the formation of a transnational community in which Brazilian and German psychiatrists provided intense scientific exchanges, especially after World War I. In Germany, Emil Kraepelin (1856-1926) gave new directions for the psychiatric research and established a model of production of the psychological and neuropsychiatric knowledge with various scientific specialties. In Brazil, as physicians Juliano Moreira (18731933) and Ulysses Vianna (1880-1935) were responsible of the reception of kraepelian's program, as well as the circulation of physicians, knowledge and institutional models across the two countries. This research highlighted that mental medicine establishes a new form of production of scientific knowledge, with emphasis on laboratory and closer to the biological and experimental bias.

Keywords

history of medicine; circulation of Kraepelin; Brazil-Germany. 


\section{Introducción}

En este artículo, estudiamos el proceso de internacionalización entre 1900 y 1914 de la medicina mental de Brasil. En este período, los médicos brasileños se acercaron a sus compañeros extranjeros, a través de viajes a Europa, conferencias y congresos internacionales, cartas y publicaciones en revistas de medicina, con el objetivo de intercambiar conocimientos, legitimar y promover sus prácticas a nivel nacional e internacional.

Como parte de un movimiento internacionalista más amplio de la medicina mental de principios del siglo XX, Juliano Moreira (1873-1933), Ulysses Vianna (1880-1935) y colaboradores acercaron la comunidad psiquiátrica de Brasil a la de Alemania. Sus acciones llevaron a la formación de una red alemano-brasileña en el campo de la medicina mental, a través de la cual circularon médicos, conocimientos y modelos institucionales, sobre todo después de la Primera Guerra Mundial (1914-1918).

El objetivo principal de este trabajo es analizar cómo y por qué se instaló un intercambio entre comunidades médicas de Brasil y Alemania. Se busca comprender los intereses que vincularon a los actores involucrados en este proceso, así como los obstáculos que tuvieron que enfrentar.

Para la producción de este artículo, la investigación histórica se basó en biografías, memorias y autobiografías, cartas, diarios de época y, sobre todo, en diversas revistas médicas y psiquátricas brasileñas y alemanas del período estudiado: Arquivos Brasileiros de Psiquiatria, Neurologia e Medicina Legal, Brasil-Médico, Gazeta Médica da Bahia y Zeitschrift für die Gesamte Neurologie und Psychiatrie.

El análisis de esos documentos históricos se realizó desde la perspectiva de la historia intelectual y la historia transnacional. Siguiendo las propuestas de Sirinelli (2003), esta historia intelectual se basó en los conceptos de generación intelectual, espacios de sociabilidad-estudiados sobre todo a través de las revistas científicas-e itinerarios políticos, analizados a través de las trayectorias intelectuales individuales y cruzadas. Sin embargo, esta investigación ha ido más allá de las propuestas de Sirinelli. Siguiendo a Latour (2000), se estudió la relación entre ámbitos locales de producción del conocimiento y la ciencia en red. A partir del concepto de comunidades epistemológicas de Peter Hass (1992) se confrontaron dos o más comunidades intelectuales, a la vez que se establecieron relaciones entre y el Estado, la política y las redes de finaciamento de la ciencia. Sin embargo, ya que este artículo se ocupa de la ciencia más allá de las fronteras nacionales, los trabajos de Crawford, Shinn y Sörlin (1992), Armitage (2004) y Clavin (2005) constituyeron un aporte fundamental para pensar la historia intelectual y de la ciencia, desde una perspectiva transnacional.

Con base en esto, se realizó una investigación sobre las trayectorias de médicos de la generación intelectual de Juliano Moreira, Ulysses Vianna y Emil Kraepelin (1856-1926), que fueron parte de una generación intelectual cuyo objetivo era crear una medicina experimental y de laboratorio, cada vez más especializada, con una orientación hacia lo biológico. La dimensión clínica se asoció a otros instrumentos de investigación y a subespecialidades. Este modelo de investigación académica y análisis clínico, montado de manera innovadora por Kraepelin y colaboradores en Múnich, circuló internacionalmente y llegó a Brasil a principios del siglo XX.

\section{Kraepelin y la Clínica de Múnich: las distintas facetas de la investigación psiquiátrica}

A menudo, la memoria de la psiquiatría orgánica (de laboratorio y experimental) se entrelaza con la historia de Emil Kraepelin. Una de las razones es que muchos psiquiatras actuales, sobre todo norteamericanos, se identificaron con su modelo de psiquiatría y se llamaron a sí mismos neo-kraepelianos. Al abordar la historia de la psiquiatría, esos psiquiatras organicistas actuales establecen relaciones directas entre ellos y Kraepelin, considerándolo un padre fundador de la psiquiatría biológica actual. Por otra parte, si bien Kraepelin no vivió durante el régimen de Hitler, se evitaron posibles continuidades con la psiquiatría alemana de los años 1930 y 1940 (Engstrom \& Weber, 2007). 
Kraepelin, de hecho, fue uno de los personajes de la generación de médicos alemanes, destacado por el desarrollo de nuevas metodologías de investigación en medicina mental, así como por la promoción de nuevas disposiciones para el trabajo clínico en varias universidades del país, desde mediados del siglo XIX. Para poder comprender cómo articuló Kraepelin su proyecto psiquiátrico y llegó a ser tan prominente en la memoria de la psiquiatría y de los psiquiatras, analizamos su formación médica y su carrera académica, en relación con la academia alemana desde Griesinger.

Wilhelm Griesinger (1817-1868) fue el primero en poner en práctica la combinación "neuropsiquiátrica" y poner en marcha la máxima de que "las enfermedades mentales son enfermedades del cerebro" (Ackerknecht, 1965, p. 2). Como profesor de psiquiatría clínica en la Universidad de Berlín, inauguró el estudio del tejido nervioso, de la anatomía del cerebro y de la patología, sin dejar de lado los problemas psicológicos ${ }^{1}$. Como gran fundador de la neurología y de la escuela neurológica de Berlín, Griesinger crea, en 1868, el Archiv für Psychiatrie und Nervenkrankheiten, primera revista médica alemana especializada en neurología (Holdorff, 2004).

En 1874, Emil Kraepelin comenzó sus estudios de medicina en un ambiente académico, lleno de los desafíos planteados por Griesinger; mostró también un gran interés en la psicología. Se puso en contacto con las lecciones de Wilhelm Wundt (1832-1920), desde el los tiempos de colegio (Kraepelin, 1987). Realizó sus estudios médicos entre 1874 y 1878, en las universidades de Leipzig y Würzburg. En este período, llevó a cabo su entrenamiento médico-psiquiátrico con Franz von Rinecker (1811-1883)² (Kraepelin, 1987).

1 Griesinger relacionó la psiquiatría con la psicología del filósofo y matemático J. F. Herbart (1776-1841) (Ackerknecht, 1965). Así como Herbart, Griesinger utilizó conceptos como neurosis e inconsciente. Según Pereira (2007), Ernst Jones afirmó que Freud hacia citas de la obra de Griesinger.

2 Franz von Rinecker, psiquiatra y dermatólogo, director de la Clínica de Psiquiatría de Würzburg, en 1863. En 1872, dirigió la clínica de la sífilis y enfermedades de la piel (Hippius, Peters, \& Ploog, 1987).
En 1878, Kraepelin obtuvo su primer trabajo como asistente Bernhard von Gudden (1824-1866) ${ }^{3}$, en la Clínica de Psiquiatría de la Universidad de Múnich. Según Spielmeyer (1927), Gudden fue uno de los más grandes patólogos del cerebro de su epoca. En Múnich, Kraepelin habría sido testigo de las dificultades y las angustias de Gudden para desenredar "el laberinto psiquiátrico" a través disecciones anatómicas (Kraepelin, 1987). Según Kraepelin (1987, pp. 16-18), en virtud de las frecuentes ausencias de Gudden y del método poco satisfactorio de preparación de las muestras, las disecciones del cerebro e investigaciones microscópicas fracasaban con frecuencia.

En el verano de 1881, la Clínica de Múnich recibió la visita del Profesor Paul Flechsig (1847-1929) 4 Según Kraepelin (1987, p. 18), había trabajado como anatomista y deseaba realizar estudios con Gudden. Fue en esta oportunidad que Kraepelin conoció a Flechsig (Shorter, 1997, p. 79).

En este contexto, de finales del siglo XIX, la psiquiatría y el hospicio pasaron por una crisis de eficacia terapéutica (Foucault, 2006), con el creciente número de pacientes y de reingresos. Muchos casos se volvían crónicos. En esta época, ya había profesores alemanes y austríacos que consideran la enfermedad mental incurable. Por eso, algunos de ellos se centraron más en la investigación científica anatómica y patológica, reservando poco tiempo o desinterés por la clínica psiquiátrica, como fue el caso Paul Flechsig, según Shorter (1997).

Con el principal objetivo de trabajar con Wundt, Kraepelin se trasladó a Leipzig y se convirtió en asistente de Flechsig. Sin embargo, su nueva experiencia de trabajo fue de corta duración. Fue destituido por el colegiado de la Universidad, después de un desacuerdo con Flechsig (Shorter, 1997). Fue acusado por Flechsig de haber cometido una falta

3 Bernhard von Gudden, profesor de psiquiatría y director del Burghoelzli, en Zurich, en 1869. Fue profesor de psiquiatría clínica en Múnich entre 1872 y 1886. También fue responsable del desarrollo del primer micrótomo para estudíos histológicos del cerebro (Hippius, Peters, \& Ploog, 1987).

4 Paul Flechsig, psiquiatra, neurólogo y profesor de psiquiatría en la Universidad de Leipzig (1877-1921). En Leipzig, publicó sus estudios sobre el mapa básico de las regiones de la corteza cerebral y sus funciones (Shorter, 1997). 
ética en el discurso oficial (Kraepelin, 1987). Con el respaldo de Gudden, Rinecker y Wundt, Kraepelin rechazó las acusaciones de Flechsig, en el Ministerio de Cultura. La desavenencia de Flechsig habría sido, precisamente, el interés de Kraepelin por la psicología wunditiana (Kraepelin, 1987) ${ }^{5}$.

Kraepelin fue una de las voces más resonantes en defensa de la psicología experimental de Wundt y de las alternativas clínicas para la anatomía patológica (Engstrom, 2004). Para él, Paul Flechsig era uno de los que no se interesaban en aprender con los pacientes y sus problemas (Shorter, 1997), a diferencia de Griesinger, quien insistió en la primacía de las observaciones y guías clínicas (Ackerknecht, 1965). Kraepelin habría sido uno de los pocos que desafió la fusión psiquiatría-neurología que se producía en las universidades prusianas (Engstrom, 2004). Con esto, nos damos cuenta de que, incluso antes de convertirse en profesor de psiquiatría, Kraepelin ya desarrollaba su posicionamiento intelectual y político en relación con las directrices de la organización psiquiátrica y su investigación académica.

En 1887, Kraepelin comenzó su carrera como profesor de psiquiatría en la Clínica de la Universidad de Dorpat (Hippius, Möller, Müller, \& Neundöfer-Kohl, 2008), cuando por fin tuvo la oportunidad de organizar un modelo de investigación psiquiátrica, que combinó la investigación clínica con la de psicología experimental. En su conferencia inaugural en Dorpart, Kraepelin habló sobre "Direcciones de la investigación psiquiátrica" (1887), dando señales sobre el proyecto de investigación en psiquiatría que quería llevar a cabo a lo largo de su trayectoria (cf., Engstrom \& Weber, 2005).

Sostuvo que la influencia de la psicosis única de Zeller ${ }^{6}$ había corrompido la investigación y la

5 Después de salir de la clínica de Leipzig, Kraepelin dijo que el laboratorio de Wundt había sido un refugio, pues trabajó allí durante unos meses (Kraepelin, 1987, pp. 21-25).

6 Ernst Albert von Zeller (1804-1877) fue director del Hospicio Winnenthal en Württemberg, donde Griesinger sirvió como asistente en la década de 1840 (Engstrom, 2004). La psicosis unitaria (Einheitspsychose) de Zeller y Griesinger se refiere a la idea de una unidad psicosomática y la existencia de una sola psicosis, pero con formas clínicas variadas, evolución y pronóstico impredecible. Griesinger, a diferencia de Zeller, optó por enfatizar la origen cerebral (Souza, 2000) observación clínica, en la década de 1860. La investigación clínica estaba casi "paralizada". Con apoyo de Wundt, Kraepelin advirtió que los investigadores no podrían poner en práctica la máxima de Griesinger ("las enfermedades mentales son enfermedades del cerebro"), si seguían con las convicciones "psicofísicas-paralelistas". Para Kraepelin, los estudios anatómicos unilaterales no mantendrían su pretensión de legitimidad científica, sin explicar "las funciones psicológicas". Esta explicación, en cambio, estaría a cargo de las investigaciones clínicas (Engstrom, 2004).

En 1890, Kraepelin regresó a Alemania par asumir la Clínica de Psiquiatría de Heidelberg. Entre 1891 y 1903, formó en Heidelberg un equipo de investigadores de renombre, médicos que permanecieron fieles a él por mucho tiempo: Alois Alzheimer (1864-1915), Gustav Aschaffenburg (1866-1944), Franz Nissl (1860-1919), Robert Gaupp (1870-1953) y Ernst Rüdin (1874-1952) (Hippius et al., 2008).

En 1903, con la muerte de Anton Bumm (18961903), Kraepelin comandó el proyecto de creación de una nueva clínica de psiquiatría, en la Universidad de Múnich, donde muchos de sus colaboradores en Heidelberg se uniron a él. Durante la construcción de la clínica, viajó a Java, con el fin de realizar sus estudios etnopsiquiáricos? ${ }^{7}$. Fue, entonces, Alois Alzheimer el responsable de organizar el montaje de los aparatos del laboratorio en Múnich (Hippius et al., 2008).

En la clínica de Múnich, Krapelin y sus colaboradores combinaron la práctica y la investigación psiquiátrica con neurología, constituyendo un modelo institucional y científico ampliamente reconocido tanto dentro como fuera de Alemania. Según Shorter (1997, p. 109), "lo que Nissl y Alzheimer podrían encontrar en sus microscopios, lo llamaron Neurología. Lo que no pudieron encontrar fue la psiquiatría". Estos dos personajes fueron entonces grandes responsables del éxito de la neurología

7 Los estudios Psiquiatría Comparada (etnopsiquiáricos) fueron realizadas por Kraepelin en varios viajes alrededor del mundo, entre ellos, en la isla de Java; allí encontró una alta frecuencia de la demencia precoz y relacionó latah e amok, respectivamente, a la histeria y a la epilepsia, con el objetivo de defender la universalidad de la enfermedad mental (Jilek, 1995). 
de Múnich que atrajo médicos de todo el mundo (Moreira, 1908).

Franz Nissl y Alois Alzheimer se conocieron en 1899, en la Clínica de Psiquiatría de la Universidad de Frankfurt. Comenzaron entonces una larga colaboración que culminó con la creación de los Histologische und histopathologische Arbeiten über die Grosshirnrinde (1904-1921). Em Frankfurt, Nissl mostró sus técnicas de coloración, su material de prueba y resultados experimentales para convencer a Alzheimer acerca de la exactitud y la importancia de los métodos neuro-histopatológicos de investigación en enfermedades psiquiátricas (Hippius et al., 2008). Nissl había creado, entonces, un "método para la examinación microscópica del sistema nervioso, de los cuales era posible, por primera vez, observar las desviaciones sutiles de la estructura normal del aparato nervioso" (Spielmeyer, 1927, p. 13).

Influenciado por Nissl, Alzheimer comenzó en Francfort sus estudios neuropatológicos (Hippius et al., 2008) ${ }^{8}$. Según Kraepelin (1922), Alzheimer, Nissl y Korbinian Brodmann (1868-1918) fueron los tres investigadores "pioneros en el campo de trabajo más importante de nostro conocimiento, lo cual debe dejar claro a nosotros la base somática para los trastornos mentales". Sobre las contribuciones de cada uno de ellos, Kraepelin afirmó:

Fue Nissl, el más grande de los tres, que, trabajando de una manera integral y constructiva, con objectivo firme de establecer las premisas para la anatomía patológica de la corteza cerebral, ayudado por todas las técnicas científicas, luchó por encontrar el plan estructural y el significado del más desarrollado tejido del cuerpo. Alzheimer, su más fiel discípulo y colaborador, luchó incansablemente, con inagotable paciencia y sacrificio para establecer, a través de un número infinito de las investigaciones individuales, los cambios corticales correspondientes a las distin-

8 Según Mauer y Mauer (2006), Alzheimer pensaba las enfermedades mentales como enfermedades del cerebro (Griesinger), en el momento en que el psicoanálisis empezó a ganar fuerza. En el congreso de psiquiatría de 1906 en Tübingen, Alzheimer presentó un trabajo sobre el caso Auguste D., primer capítulo de la enfermedad de Alzheimer. Sin embargo, su trabajo fue recibido con poco interés por sus colegas. tas formas de trastorno mental, (...) permitiendo al clínico (...) pruebar sus hipótesis y hacer (...) de la medicina el más poderoso instrumento de progreso. Brodmann [ $\left.{ }^{9}\right]$ había fijado la tarea de descubrir la división celular en la corteza y así preparó el terreno para el trabajo futuro que debe informar a nosotros la localización y evolución de la enfermedad en la corteza y la extensión de áreas de tejidos individualmente atacados por ella. (Kraepelin, 1922, p. 91)

La presencia de Nissl y Alzheimer en la equipo de investigación y enseñanza permitió la formación y capacitación de nuevos investigadores, a través de la transferencia de técnicas y metodologías esenciales para el trabajo de laboratorio. Este proceso garantizaba la renovación de científicos en la clínica de Múnich. Por eso, jóvenes neurólogos atraídos por la clínica, se encontraban allí por esos años: Eugen Kahn (1887-1973), Felix Plaut (1877-1940), Max Isserlin (1879-1941), Walter Spielmeyer (1879. 1935), Alfons Maria Jakob (1884-1931), Hugo Spatz (18888-1969) y Julius Hallervorden (1882-1965), entre otros (Hippius et al., 2008).

Hacía tiempo que muchos médicos alemanes luchaban por encontrar la base física y somática de la enfermedad mental, para poner en práctica las premisas de Griesinger. Com esta finalidad, Nissl y Alzheimer realizarán grandes hallazgos y contribuiciones para la ciencia alemana, por lo cual fueron claves para el éxito de la Clínica Múnich, dentro y fuera de Alemania.

Edward Shorter (1997, p. 66) considera a Kraepelin "por lejos, el más prominente de los psiquiatras académicos alemanes" de su tiempo. Pero, al naturalizar el tamaño del reconocimiento recibido por Kraepelin a lo largo de su trayectoria, Shorter no explicó cómo fue eso posible. La afirmación carece de cuestionamiento mayor. La localización cerebral de la enfermedad mental era un viejo sueño y era entendida como un pasaporte de ingreso en el "hall de las ciencias". Permitiría, en la opinión de los mé-

9 Fue en Berlín, en la Neurologische Zentralstation (embrión del Instituto Kaiser Wilhelm de Investigaciones del Cerebro), donde Brodmann desarrolló sus estudios del mapa cortical (topografía histológica) (Holdorff, 2004). Trabajó en el equipo de Kraepelin en 1918, poco antes de morir. 
dicos de la época, que la medicina mental alcanzara el camino de la bacteriología, dentro del proceso de equiparar la medicina (pensada como fisiología experimental) con las ciencias físicoquímicas, como deseaba Claude Bernard, en 1865 ${ }^{10}$. iEntonces, por qué fue el nombre de Kraepelin y no el de Nissl o de Alzheimer ${ }^{11}$ el más destacado de esta generación?

Como hemos visto, Kraepelin siguió un camino distinto al de los psiquiatras anatomo-patólogos de su tiempo. No consideraba la clínica como algo menor. Él era ante todo un clínico. Más allá del reconocimiento obtenido, en sus escritos y estudios personales, como el Tratado de psicopatología (Die Psychiatrie) ${ }^{12}$, o incluso los estudios en psiquiatría comparada y psicología experimental, es necesario aún entender su proyecto institucional para explicar su éxito local e internacional.

En los hospicios, Kraepelin introdujo estudios longitudinales y mapas genealógicos y estadísticos con los docentes (Zahlkarten), para perfeccionar el diagnóstico e identificar precozmente las patologías. Además, fue muy exitoso en la implementación de un nuevo modelo institucional para la psiquiatría, lo que amplió el alcance de la investigación psiquiátrica, por medio de laboratorios interconectados que incorporaban diversas ciencias auxiliares (Spielmeyer, 1927). Esto fue posible también gracias a los trabajos realizados en conjunto con sus destacados colaboradores, como Nissl y Alzheimer. Por lo tanto, Kraepelin fue un gran articulador y tuvo la cualidad de organizar un equipo altamente calificado.

Nuestra hipótesis es que Kraepelin ejerceió un tipo menos autocrático de mando, focalizándose en la motivación de los investigadores, a través de la delegación de funciones y responsabilidades. De esta manera, siguió a Wilhelm Humboldt en

10 Véase Bernard (1944).

11 Nissl y Alzheimer se convierten en profesores catedráticos de psiquiatría en otras universidades alemanas (Engstrom, 2004), pero no alcanzarán a repetir o superar el éxito de Kraepelin.

12 De todas las publicaciones de Kraepelin, su trabajo con la máxima expresión fue Die Psychiatrie, tratado de psiquiatría que alcanzó nueve ediciones y llegó a contener 2.500 páginas, entre las ediciones de 1883 hasta 1913. La novena y última edición fue escrita con Johannes Lange y publicada solo en 1927, ya después de la muerte de Kraepelin (Hippius et al., 2008). su concepción de la universidad, así como de la docencia y la investigación, quien afirmó que "la actividad intelectual sólo progresa cuando hay cooperación, no sólo para que un investigador proporcione lo que falta al otro, sino también para estimular el otro" (Humboldt, 1997). Con este ideal, Kraepelin organizó una investigación multifacética en Múnich, ampliamente reconocida por sus colegas germánicos y extranjeros.

Además de la neurología (neuropatología, neurohistologia y neuroinmunología), Kraepelin y sus colaboradores ampliaron la investigación psiquiátrica -sin olvidar la importancia de la clínica- con estudios en psicología experimental, psicofisiología, psiquiatría forense, farmacopsiquiatría y farmacopsicología, psiquiatría transcultural, epidemiología psiquiátrica y psiquiatría genética (Kraepelin, Hippius, Peters, Ploog, Hoff, $\&$ Kreuther, 1987). Pero eso fue un proyecto de años. En 1904, había en Múnich apenas los siguientes laboratorios: Laboratorio de Psicología Experimental, Laboratorio de Neuropatología, Laboratorio Químico (Hippius et al., 2008). El Laboratorio de Psicología Experimental había sido un antiguo deseo de Kraepelin y era dirigido por él. Los estudios en este laboratorio resultaron en una publicación de ocho volúmenes, en 1910, con el título de Estudios psicológicos (Psychologische Arbeiten).

El Laboratorio de Neuropatología (donde se realizaban análisis microscópicos) fue dirigido por Alzheimer hasta 1912 y después por Walter Spielmeyer ${ }^{13}$; y el Laboratorio Químico fue dirigido inicialmente por Erwin Rohde -colaborador de Kraepelin desde Heidelberg. Entre 1908 y 1909, este laboratorio fue administrado por Fritz Lotmar y en 1910 por Rudolf Aller (1883-1963). Cabe aún destacar que posteriormente fueron creados un archivo de casos clínicos y otros dos laboratorios: el Laboratorio Genealógico Demográfico y el Laboratorio de Sorología, donde trabajarán,

13 Walter Spielmeyer, neurohistopatologista, médico asistente de Alois Alzheimer y colaborador de Kraepelin desde 1912, entre 1926 y 1931, fue director del Instituto Alemán para la Investigación Psiquiátrica en Múnich, hoy Instituto Max-Planck de Psiquiatría (Weber, 2000). 
respectivamente, Ernst Rüdin ${ }^{14}$ y Felix Plaut $^{15}$ (Hippius et al., 2008).

\section{La trayectoria de Kraepelin y de la psiquiatría alemana en Brasil: la formación de una comunidad alemano- brasileña en la medicina mental}

La medicina enseñada en la Facultad de Medicina de Río de Janeiro y practicada en el Hospicio de Pedro II, en el siglo XIX, tenía como característica un fuerte diálogo con médicos franceses. Al comienzo del siglo XX, la medicina alemana ya era utilizada en la Facultad de Medicina y en el Hospital Nacional (antiguo Hospicio de Pedro II) (Magalhães, 1932). En el caso de hospicio nacional, el alienismo francés, hasta entonces predominante, perdió la hegemonía y pasó a competir con la psiquiatría alemana, especialmente, con el modelo defendido por Emil Kraepelin (Facchinetti \& Muñoz, 2013).

La trayectoria de Kraepelin en Brasil se remonta al período en que Juliano Moreira (1873-1933) era profesor en la Facultad de Medicina de Bahia y, principalmente, cuando trabajó en Río de Janeiro como Director del Hospicio Nacional (1903 y 1930) y como director de la Asistencia Médico-Legal para Enfermos Mentales (Venâncio, 2003). A fines de la década de 1890, Moreira ganó una beca de estudios y realizó cursos con Paul Flechsig (1847-1929), Emil Kraepelin, Valentin Magnan (1835-1916), Rudolph Virchow (1821-1902) y Richard von Kraft-Ebing (1840-1902) (Passos, 1975).

En 1900, Moreira regresó a Alemania y visitó varias clínicas psiquiátricas (Moreira, 1908). A lo largo de siete publicaciones, de 1901 a 1902, divulgó en la Revista do Grêmio dos Internos dos Hospitais

14 Ernst Rüdin, psiquiatra suizo, genetista e higienista racial, fundó la Sociedad Alemana de Higiene Racial, con Alfred Ploetz (1860. 1940) en 1904. Dos años más tarde, trabajó con Kraepelin en Múnich. Entre 1931 y 1945, dirigió el Instituto Alemán para la Investigación Psiquiátrica. En su trayectoria, estuvo muy cerca de la higiene racial alemana y del régimen de los nazis (Weber, 1996).

15 Felix Plaut, neurólogo y neuroinmunólogo de origen judío, estudió con el médico y bacteriólogo August von Wassermann (18661925). Hizo parte del equipo de Kraepelin en Múnich desde 1904. Durante el nazismo, fue despedido por Rüdin en 1935 (Hippius et al., 2008). sus impresiones acerca de las clínicas psiquiátricas de Leipzig, Halle y Würzburg. Acerca de Leipzig, exaltó los cursos de Wundt, así como la importancia histórica de la clínica de psiquiatría dirigida por Paul Flechsig. También describió sus instalaciones físicas, los cursos de Fleschig y la trayectoria de este médico alemán (Moreira, 1901a, 1901b).

Entre los aspectos de la psiquiatría alemana, Moreira se entusiasmó con las investigaciones de laboratorio. En 1902, publicó un artículo para defender la necesidad del establecimiento de laboratorios en los hospitales, en Brasil:

No es sin cierta vergüenza que me propongo abordar el asunto indicado en el título por encima de estas líneas. (...) Dondequiera que la civilización ha penetrado (...) ya no es necesario predicar los beneficios de la organización de los laboratorios en los hospitales. Lamentablemente nosotros necesitamos todavía de una gran propaganda a favor de la fundación de laboratorios en diversos servicios hospitalarios del país. (...) En el correr de las 7 $7^{\underline{a}}$ y $8^{\underline{a}}$ décadas son aportaciones al estudio de diagnóstico físico, especialmente en lo que respecta a la auscultación y percusión, que llenan las páginas de revistas profesionales. Diagnóstico físico, en el sentido estricto de la frase, fue llevado a sus más íntimos detalles, siempre en comparación con los fenómenos clínicos y los resultados de las necropsias. Además el plexímetro y el estetoscopio, termómetro y el microscopio, todavía imperfecto, y en casos raros el esfigmógrafo comenzarán a proporcionar servicios clínicos (...). (Moreira, 1902, pp. 439-441)

Moreira (1902) también destacó los avances en el estudio de la patología del metabolismo y estudios de modificaciones químicas de la sangre y de las secreciones. Junto a los avances de la química clínica (1902, p. 443), señaló que la bacteriología había salido "triunfante" con las aportaciones de Robert Koch y que sería una guía segura en la diferenciación etiológica de enfermedades. Concluyó que los logros en el campo médico tenían su origen en las investigaciones de laboratorio. Por lo tanto, a pesar de las declaraciones sobre el gran costo de la instalación de laboratorios, argumentó que un buen 
servicio clínico debe tener "un instituto o un laboratorio de patología; un laboratorio de bacteriología y uno de química clínica" (Moreira, 1902, p. 446).

En virtud de los viajes de Moreira a Alemania, intercambió cartas en 1905 con Kraepelin (Moreira, 1905a, 1905b), quien había mostrado interés en viajar a Brasil para obtener datos sobre patologías en indígenas brasileños, como parte de su agenda de investigación en psiquiatría comparada. Dicho viaje no se llevó a cabo, porque Moreira respondió que no había indígenas puros en un hospicio nacional.

Entre 1906 y 1907, Juliano Moreira regresó a Alemania. Sus impresiones fueron el tema de su conferencia, en 1907, en la Academia Nacional de Medicina, así como un artículo sobre la psiquiatría de Múnich. Según Moreira, la clínica de Múnich del profesor Emil Kraepelin tenía modernos laboratorios y instalaciones, además de cursos que médicos de diversas partes del mundo se disputaban: "en el curso normal del verano en el año 1906, me vio en el anfiteatro de los oyentes de la clínica de Múnich de las más variadas nacionalidades, muchos de los cuales ya llevan un nombre como alienistas" (Moreira, 1908, p. 184). Aunque tenía mucha empatía por Kraepelin y su programa de investigación y enseñanza, no podemos resumir su proyecto profesional a la difusión de Kraepelin en Brasil. Tres años después de visitar Alemania, declaró abiertamente que había "hecho esfuerzos para popularizar la beneficiosa influencia ejercida por 20 clínicas alemanas sobre el estudio de enfermedades mentales" (Moreira, 1910, p. 376). Moreira era un experto conocedor de la psiquiatría alemana, pero también estaba actualizado en relación con las más modernas instalaciones hospitalarias y modelos institucionales de Europa occidental:

Es considerable la variedad de tipos de pabellones que he visto adoptarse en varios asilos colonias de Europa. La experiencia de los más notables alienistas demostró en Escocia, en Suiza, en Alemania, en Bélgica, en Holanda, etc., que, al menos, la mitad de los alienados, a menudo dos terceras partes, pueden disfrutar de cierta libertad. Así que es perverso dejar en 100 pacientes, 66 sometidos a estricto secuestro, cuando sólo 33 de esos lo necesitan. Y eso es lo que necesariamente no ocurre a quien condena la puerta abierta debido a los posibles peligros de la libertad de los pacientes a sí mismos y a la seguridad pública. (p. 380)

Este discurso nos ayuda a entender por lo menos dos cuestiones importantes: por un lado, las referencias que Moreira utilizó para apoyar el largo proceso de reforma en la estructura física del Hospicio Nacional y en la Asistencia a los Alienados, entre 1903 y 1930. Por otro lado, hace explícita la resistencia al cambio de los paradigmas psiquiátricos brasileños -sin mencionar las dificultades en la obtención de fondos por parte del Gobierno, como destacó en 1902 en su artículo sobre la necesidad de laboratorios ${ }^{16}$.

Con el amplio proceso de reforma iniciado en 1903, inspirado en la psiquiatría alemana, Moreira eliminó las rejas de hierro del manicomio y las camisas de fuerza. Introdujo la clinoterapia y el uso de baños en el tratamiento de los enfermos mentales y fue responsable también de aceptar la admisión voluntaria de pacientes. En su administración en el hospicio, se crearon laboratorios y pabellones especializados, además del sistema de dispensarios. Con la creación del laboratorio anatomo-patológico (Engel, 2001), en los primeros años de la administración, "las punciones lumbares fueron practicadas con regularidad y el examen citológico del líquido cerebroespinal apoyó y aclaró los diagnósticos" del hospicio, desde 1906 (Dunningham, 2008, p. 73).

Además del laboratorio anatomo-patológico del Hospicio Nacional, donde se realizaron diversos exámenes clínicos, durante la gestión de Moreira se inauguraron otros laboratorios, como el Laboratorio Nissl, en la sección Pinel, y el laboratorio del Ambulatorio Graffée-Guinle que funciona en conjunto con el dispensario Afrânio Peixoto. Este laboratorio era "especializado en la investigación del germen de lues y sus determinaciones morbosas", y en cuanto a la Asistencia a los Alienados, las

16 No fue por casualidad que muchos psiquiatras hiceran carrera política. Entre ellos, Teixeira Brandão, Antônio Austregésilo y Afrânio Peixoto (Engel, 2001). 
colonias de Jacarepaguá y Engenho de Dentro, así como el Instituto de Neuropatología y el Manicomio Judiciario del Distrito Federal también tuvieron laboratorios ("Hospital Nacional de Alienados Comemora”, 1925).

A partir de todo lo anterior, es posible darse cuenta de la importancia de Juliano Moreira en el establecimiento de las relaciones científicas en medicina mental entre Brasil y Alemania. Además de los viajes científicos y las visitas a las instituciones europeas, Moreira participó de varias conferencias internacionales y estableció un estrecho vínculo con los médicos extranjeros (Facchinetti \& Muñoz, 2013), ganando poco a poco el reconocimiento de sus pares en Europa. Junto con Afrânio Peixoto ${ }^{17}$, hizo parte del The International Committee for the Study of the Causes and Prophylaxis of Mental Disease, como uno de los 27 miembros de distintas nacionalidades que se reunieron por primera vez en la Universidad de Amsterdam, el 4 de septiembre de 1907 (Smith, 1910).

Por otra parte, desde los principios de 1900, Moreira buscó difundir internacionalmente los avances de la psiquiatría brasileña y así dar mayor visibilidad a las instituciones de su país. En 1909, el The British Journal of Psychiatry publicó un artículo suyo sobre los avances de la psiquiatría en Brasil (Moreira, 1909), donde señaló que los progresos realizados en psiquiatría por las repúblicas de Sudamérica eran desconocidos por los europeos. Luego, recordó todos los pasos de las reformas llevadas a cabo por él, entre 1903-1909, en la legislación y en el Hospicio Nacional-de las cuales ya hemos hecho referencia-. Por último, presentó los avances de la psiquiatría de São Paulo y los principales temas y ponencias presentadas por sus colegas de Brasil y de los demás países de América Latina en la sección de Psiquiatría, Neurología y Medicina Legal

17 Julio Afrânio Peixoto (1876-1947), médico que colaboró intensamente con Moreira en el Hospital Nacional de Alienados para las reformas de 1903 y 1904. En 1907, fue profesor de medicina legal en la Facultad de Medicina de Río de Janeiro y presidente de la Academia Brasileña de Letras (1922-1923). También fue elegido diputado federal de Bahía, con dos períodos consecutivos (1924-1930) y rector de la Universidad del Distrito Federal (1935) (Ribeiro, 1950). del IV Congreso Internacional Latinoamericano de Medicina.

En Brasil, así como hizo Kraepelin en Alemania, Moreira también se acercó a la psicología, a la psiquiatría comparada y a la investigación experimental, además de defender la especialización dentro de la medicina mental brasileña, y defendió la universalidad de la enfermedad mental. Se opuso también al determinismo climático y racial muchas veces utilizado para explicar las enfermedades de los brasileños y condenar la misigenación del pueblo, así como la viabilidad de Brasil como nación moderna. Para Moreira, como para Kraepelin, la degeneración era parte de un problema epidemiológico, resultante de las condiciones de salud, de la sífilis, del alcohol y de la mala educación (Engstrom, Burgmair, \& Weber 2002; Facchinetti \& Muñoz, 2013).

Reunió también un equipo de reconocidos médicos calificados para trabajar en el Hospicio Nacional: Miguel Pereira (1871-1918), Afrânio Peixoto, Antônio Austregésilo (1876-1960), Fernandes Figueira (1863-1928), Raul Leitão da Cunha (18811947), Álvaro de Andrade Ramos, Humberto Neto Gotuzzo, entre otros (Facchinetti \& Muñoz, 2013). Fue así como Moreira ganó aliados en el proceso de difusión de los nuevos criterios para la psiquiatría.

Al descentrar nuestra mirada de la figura de Moreira, es posible identificar la importancia de otros médicos en el establecimiento de las relaciones con Alemania. En 1905, Antônio Austregésilo hizo la traducción del texto intitulado "Paranoia" (Verrückheit), que Kraepelin escribió en su libro llamado Psiquiatría (Die Psychiatrie) (Kraepelin, 1905). Así como Moreira y Peixoto (1905), Ulysses Vianna ${ }^{18}$ también entendió la paranoia a la manera de Kraepelin (Carrilho, 1935), en oposición a los médicos Henrique Roxo ${ }^{19}$ (1877-1969) y Teixeira

18 Ulysses Machado Pereira Vianna Filho fue a partir de 1905 asistente voluntario y depués interino del Hospicio Nacional. Participó en un concurso público para la misma instituición e ingresó como médico de la Sección Pinel, de pacientes masculinos (1908-1912) (Carrilho, 1935; Piccinini \& Bueno, 2008).

19 Henrique de Brito Belford Roxo fue médico asistente de la Clínica de Psiquiatría de la Facultad de Medicina de Rio de Janeiro y del Pabellón de Observaciones, entre 1904 y 1921. Remplazó varias 
Brandão ${ }^{20}$ (1854-1921) (Facchinetti \& Muñoz, 2013).

En colaboración con Afrânio Peixoto, Moreira publicó el artículo titulado "Classificação de moléstias mentais do professor Emil Kraepelin", en el cual señalaron la trayectoria del psiquiatra alemán y comentaron los quince grupos de su clasificación psiquiátrica (Moreira \& Peixoto, 1905, p. 205). En 1910, Moreira, Peixoto y colaboradores aprobaron, después de casi tres años de largos debates, un modelo clasificatorio nacional bajo las bases kraepelianas, aunque con la incorporación de referencias de la psiquiatría francesa, como deseaba Henrique Roxo (Muñoz \& Facchinetti, 2011).

Los logros de Moreira al divulgar la psiquiatría alemana en Brasil atrajeron médicos brasileños a Alemania. En 1912, el Dr. Ulysses Vianna, que había realizado cursos en Tübingen, en la clínica de los eminentes profesores Matzenbacher y Robert Gaupp, decidió viajar a Alemania, estableciéndose en Múnich para tomar cursos con Kraepelin. Su objetivo era "perfeccionarse en los asuntos de su especialidad" ("Vida Social: viajantes", 1912). De su primer paso por Alemania, surgió una tesis médica sobre el diagnóstico de la arterioesclerosis cerebral, donde se combinaba el estudio clínico con el estudio histopatológico (Vianna, 1911). Con esa tesis, obtuvo el título de "livre-docente" (Carilho, 1935).

El segundo viaje de Vianna a Alemania tuvo una mayor duración. Esta vez, trabajó como asistente de Alzheimer, en Múnich. Cuando este fue designado para la Cátedra de Psiquiatría de Breslau, en 1913 (Hippius et al., 2008), Vianna lo acompañó (Bueno \& Piccini, 2008). En ese mismo año, Moreira y Vianna (1913) publicaron un artículo sobre la parálisis progresiva, en una importante revista alemana de medicina mental, Zeitschrift für die Gesamte Neurologie und Psychiatrie, que contaba con

veces Teixeira Brandão en la dirección del Pabellón de Observaciones (Muñoz, Facchinetti, \& Dias, 2011).

20 Teixeira Brandão fue profesor de psiquiatría y enfermedades nerviosas, entre 1883 y 1921. Además, fue director del Hospicio Nacional y de la Asistencia Médico-Legal para Enfermos Mentales (1890-1899), director del Pabellón de Observaciones, entre 1894-1921 y diputado federal (1904-1907 y 1911-1921) (Facchinetti \& Muñoz, 2013)
Alzheimer, Gaupp, Williams y Lewandosky ${ }^{21}$, en su editorial. El artículo, cuyo tema era de gran interés de los médicos alemanes, no debe tomarse como una sola publicación. Ejemplifica la importancia de Ulysses Vianna como interlocutor en el proceso de formación de la comunidad alemano-brasileña en medicina mental.

Con la Primera Guerra Mundial, Vianna decidió regresar a Río de Janeiro y empezó a divulgar el trabajo de Alzheimer en Sudamérica (Piccinini \& Bueno, 2008). En el Hospicio Nacional, Vianna fundó y dirigió el Laboratorio Nissl -que sostuvo con sus propios recursos-, donde llevó a cabo investigaciones en neuropatología y histopatología del sistema nervioso, continuando así con los estudios realizados en Alemania. Desde entonces, se convirtió en un divulgador de la medicina mental alemana y mantuvo estrechos lazos con los médicos alemanes (Carrilho, 1935).

Con Arthur Moses (1886-1967)22, Vianna realizó grandes esfuerzos en el estudio de la serología de la sífilis y de la reacción de Wassermann en psiquiatría, contribuyendo a la difusión del trabajo del médico alemán August von Wassermann, en Brasil (Moses, 1909; Teive, Almeida, \& Werneck, 2006). La asociación entre Vianna y Moses fue de gran importancia para fortalecer las relaciones entre el Hospicio Nacional y el Instituto Oswaldo Cruz, así como para el proceso de divulgación de la ciencia alemana en Brasil. Además de dominar el alemán, mantuvieron contacto frecuente con instituciones científicas de ese país. En 1928, Moreira, Vianna y Moses fueron premiados con la medalla de oro de la Universidad de Hamburgo (Boletim da Sociedade Brasileira de Neurologia, Psiquiatria e Medicina Legal, 1929, p. 54).

Estos tres personajes, junto con Henrique da Rocha Lima (1879-1956) ${ }^{23}$, fueron centrales para

21 Sobre esos médicos alemanes, ver Engstrom (2004).

22 Arthur Moses, médico y científico del Instituto Oswaldo Cruz (1908-1917). En 1917, trabajó en el Ministerio de Agricultura. También fue miembro de la Academia Brasileña de Ciencias (Teive, Almeida, \& Wernerck, 2006)

23 Henrique da Rocha Lima, microbiólogo brasileño, trabajó en el Instituto Soroterápico del Distrito Federal (que, en 1908, había sido rebautizado como Instituto Oswaldo Cruz), entre 1903 y 1909. Fue investigador en el Instituto de Enfermedades Maríti- 
la formación de la comunidad médica alemanobrasileña. De esta manera, se evidencia la magnitud de las redes de contactos de Moreira y Vianna para el desarrollo y la difusión de la ciencia brasileña en el extranjero y para la divulgación de la ciencia alemana en Brasil. Esas redes incluyeron fuentes estatales de financiación y alianzas con otras instituciones nacionales y estranjeras, así como con otras especialidades médicas.

Sin embargo, la recepción de Kraepelin y de la medicina mental alemana en Brasil se llevó a cabo en medio de una serie de resistencias ${ }^{24}$ del grupo formado Teixeira Brandão, Marcio Nery (18651911) ${ }^{25}$ y Henrique Roxo, médicos responsables de la clínica de psiquiatría y enfermedades nerviosas en la Facultad de Medicina de Río de Janeiro, que funcionaba en el Pabellón de Observaciones ${ }^{26}$.

Según Antônio Austregésilo (1906), Henrique Roxo, que era "un discípulo del profesor Teixeira Brandão", adoptó, en su libro Lecciones de psiquiatría y enfermedades nerviosas, de 1906, una clasificación centrada en las ideas de su maestro, solo divergía en "raros puntos doctrinales". En lo referente a la clasificación de Kraepelin, Roxo ya había presentado varias contrapropuestas: para tratar la demencia, "no aceptada como Kraepelin la concebida"; sobre la inclusión de la forma paranoide en la psicosis progresiva crónica sistematizada, pensaba que era "una audacia de Kraepelin". De acuerdo con otros autores brasileños, como Brandão y con médicos franceses, Roxo admitió la confusión mental, aunque con restricciones y "se opuso a la manera del pensamiento de Kraepelin”. Concebía la paranoia como originaria, cuyo delírio surgiría "d'emblée",

mas y Tropicales de Hamburgo, entre 1909 y 1927. En 1919, fue nombrado profesor en la Universidad de Hamburgo (Silva, 2010).

24 Si dentro de la psiquiatría, Moreira y Brandao son representantes de perspectivas teóricas distintas, hay que destacar que estos médicos defendieron algunos objetivos similares, como por ejemplo mayor financiación para la psiquiatría, en legislativo nacional (ver Engel, 2001).

25 Márcio Filafiano Nery, director interino del Hospicio Nacional (1898-1899), profesor adjunto de psiquiatría en la Facultad de Medicina de Río de Janeiro (1904-1911) (Facchinetti \& Muñoz, 2013).

26 Institución de enseñanza y evaluación de pacientes, que se quedaba en el Hospicio Nacional de Alienados. Sobre el Pabellón de Observaciones, ver Muñoz, Facchinetti, y Dias (2011). contrariamente a las estadísticas de Kraepelin (Austregésilo, 1906, p. 394).

En 1913, con fondos de la Facultad de Medicina de Río de Janeiro, Henrique Roxo también viajó a Europa. En su informe de viaje a la Facultad de Medicina (1913), agradeció la oportunidad informarse de los progresos en psiquiatría. Afirmó haber estudiado detalladamente la psiquiatría francesa y alemana, porque estas dos "escuelas antagónicas" habrían propiciado el avance en el estudio de la psiquiatría. Sobre su experiencia en Francia, señaló que:

El método de enseñanza es totalmente diferente a lo que se observa entre nosotros (...). Es un sistema más colegiado. El profesor presenta un enfermo y llama a dos estudiantes para examinar. Hace los análisis sobre lo que verificaron y realiza durante quince minutos comentarios sobre la entidad clínica. (...) Al final de algunas clases, presenta proyecciones de muestras de histología patológica o cintas cinematográficas de pacientes con demencia precoz, de maníacosdepresivos, etc. El diagnóstico fue confirmado con seguridad, utilizando los recursos de laboratorio. La clínica, con eso, está ricamente instalada. Hay vastas habitaciones de química biológica, anatomía patológica, histología patológica y, sólo para la psicología, había 4 gabinetes. No fue una sola vez que vi el profesor hacer el diagnóstico diferencial entre la imbecilidad y la demencia precoz, por ejemplo, sin que la pesquisa de asociación de ideas sacase las dudas. (Roxo, 1913, pp. 513-514)

Sobre Berlín, Roxo señaló que en esa ciudad la clínica de psiquiatría y enfermedades nerviosas trabajaban en una pequeña casa de campo, cada una con un ala. También habría una clínica ambulatoria de enfermedades nerviosas; así como en Múnich, se utilizaban los baños en el tratamiento de los pacientes y recursos de laboratorio para realizar exámenes y elucidar el diagnóstico. En la clínica psiquiátrica de Königlische Charité, Roxo presenció la práctica de la helioterapia (es decir, el "moderno tratamiento por la exposición al sol") y electroterapia, bajo la "forma de la franklinisación [sic.] y alta frecuencia", diferente de la clínica de Múnich y las 
colonias de Eglfing y Haar, donde no había tales recursos terapéuticos. El uso de medicación sería más frecuente en Berlín que en Múnich. Las clases en Berlín también tenían recursos de proyección, inexistentes en Brasi ${ }^{27}$. Comparando las clínicas de Berlín y Múnich con la de París, Roxo señaló que en Alemania la psiquiatría estaba dotada de más recursos de laboratorio. Además,

(...) tanto en Francia como en Alemania, vio en gran escala la práctica del reposo en cama. Hay una preocupación constante para considerar a la persona que sufre el cerebro como sufriendo de cualquier otras vísceras. Del mismo modo, la íntima relación que existe entre el sistema nervioso y otras vísceras induce a investigarse las tóxicos-infecciones generales, que pueden causar los síndromes mentales. El diagnóstico es siempre robustecido por los datos de la psicología y química biológica. (Roxo, 1913, p. 504)

Roxo elogió el proceso de diagnóstico europeo, con amplio uso de los recursos de laboratorio y la competencia de los asistentes. Sin embargo, era crítico de los esfuerzos terapéuticos: "la fuerza de querer simplificar la terapia, van al extremo de casi nada prescribir". Para Roxo, el secreto de la prosperidad en Europa sería la especialización de áreas de investigación y tareas (Roxo, 1913, p. 515).

A pesar de ser admirador de la psiquiatría francesa, Roxo realizó un analisis crítico de la organización psiquiátrica en Europa como un todo. Sin embargo, tuvo que reconocer la importancia de los avances logrados por los europeos, principalmente franceses y alemanes, ejemplo del gran trabajo con los recursos de laboratorio. No obstante, el análisis de Roxo se interpretó como una defensa, en muchos momentos nacionalista, de los psiquiatras y

$27 \mathrm{Al}$ criticar la falta de recursos de proyección en la Clínica de FMRJ, que Teixeira Brandão ya había pedido, Roxo señaló que "la escasez de recursos materiales se sentía a cada paso y el esfuerzo del profesor era enorme para evitar que su enseñanza se vuelva imperfecta y defectuosa. En Europa, diferente de acá, nadie entiende que debemos economizar en la educación" (Roxo, 1913, p. 505). profesores de la psiquiatría brasileños que, según él, superaban las dificultades de la falta de recursos y problemas de infraestructura.

Además, Henrique Roxo fue un personaje que hizo muchas críticas a la clasificación de Kraepelin, defendiendo las concepciones de Teixeira Brandão, Marcio Nery y de los médicos franceses Valentin Magnan y Emmanuel Régis (1855-1918) (Muñoz \& Facchinetti, 2011). Aunque mantenía sus diferencias teóricas en relación con los médicos alemanes, tuvo progresivo interés por los modelos institucionales, sobre todo, por la investigación científica y de laboratorio de la medicina mental germánica.

\section{Conclusión}

En el presente artículo, se defiende la idea de que el período anterior a la Primera Guerra Mundial marca el inicio del diálogo entre la psiquiatría brasileña y la alemana. En ese momento, constituían una comunidad médica transnacional en medicina mental que estrechaba el intercambio entre los dos países. A diferencia de lo que ocurrió después de la guerra, la relación entre los médicos de los dos países tuvo un carácter más unilateral, con grandes esfuerzos realizados por los médicos brasileños para acercarse a sus colegas alemanes, a menudo con viajes a Europa. Sin embargo, la documentación muestra que, aunque Moreira ya tenía reconocimento entre los médicos alemanes y europeos, en general, parecían saber poco sobre la medicina mental de Brasil.

Además, entre 1900 y 1914, se observa que la comunidad psiquiátrica brasileña empezaba a conseguir recursos públicos para inversiones locales y para financiar viajes a Europa, a través del Ministério de Justicia y Negócio Interiores -entre la que estaban la Facultad de Medicina, la Asistencia a los Alienados y otras instituciones psiquiátricas-. Aún quedan muchas preguntas sobre la relación entre la comunidad psiquiátrica y el Estado. Cabe señalar que esos psiquiatras convivían con la falta de recursos, como destacó Henrique Roxo (1913), y con muchas limitaciones en la infraestructura de los hospicios, que se manifestaban en especial en la superpoblación. La lucha por la reforma y por más recursos, así como las críticas a la psiquiatría y a los 
hospicios, condujeron a muchos médicos hacia la carrera política, como fueron los casos de Teixeira Brandão, Austregésilo y Peixoto en Brasil. Pero esto es tema para trabajos posteriores.

\section{Referencias}

Ackerknecht, E. H. (1965). [Prefacio]. En W. Griesinger, Mental pathology and therapeutics. New York: Hafner. (Reimpreso)

Armitage, D. (2004). The fifty year's rift: Intellectual history and international relations. Modern Intellectual History, 1(1), 97-109.

Austregésilo, A. (1906). Dr. Henrique Roxo: Lições de Psiquiatria e Moléstias Nervosas - Rio, 1906. Arquivos Brasileiros de Psiquiatria, Neurologia e Ciências Afins, ano II, n. 4, p. 393-396.

Bernard, C. (1944). Introducción al estudio de la medicina experimental. Buenos Aires: EMECE Editores.

Boletim Da Sociedade Brasileira De Neurologia, Psiquiatria E Medicina Legal. (8 de abril de 1929). Arquivos Brasileiros de Neuriatria e Psiquiatria, 11(1), 54.

Calvin, P. (2005). Defining transnationalism. Contemporary European History, 14(4), 421-439.

Carrilho, H. (1935). Noticiário: Professor Ulysses Vianna. Arquivos do Manicômio Judiciário, 6(1-2), 115 130.

Crawford, E., Shinn, T., \& Sörlin, S. (1992). Denationalizing science: The contexts of international scientific practice. Sociology of the Sciences. Dordrecht/Boston/ London: Kluwer Academic Publishers.

Dunningham, W. (2008). A. Juliano Moreira: notas sobre a sua vida e obra. Gazeta Médica da Bahia, 78(1), 72-75.

Engel, M. G. (2001). Os delírios da razão: médicos, loucos e hospícios, Rio de Janeiro 1830-1930. Rio de Janeiro: Editora Fiocruz.

Engstrom, E. J. (2004). Clinical psychiatry in Imperial Germany: A history of psychiatric practice. New York: Cornell University Press.

Engstrom, E. J., Burgmair, W., \& Weber, M. M. (2002). Emil Kraepelin's 'self-assessment': Clinical autography in historical context. History of Psychiatry, 13(49), 89-98.
Engstrom, E. J., \& Weber, M. M. (2005). The directions of psychiatric research by Emil Kraepelin. History of Psychiatry, 16(3), 345-349.

Engstrom, E. J., \& Weber, M. M. (2007). Making Kraepelin history: A great instauration? History of Psychiatry, 18(3), 267-273.

Facchinetti, C., \& Muñoz, P. (2013). Emil Kraepelin na ciência psiquiátrica do Rio de Janeiro, 1903-1933. História, Ciências, Saúde - Manguinhos, 20(1), 239-262.

Foucault, M. (2006). O Poder psiquiátrico. São Paulo: Martins Fontes.

Haas, P. (1992). Introduction: Epistemic communities and international policy coordination. International Organization, 46(1), 1-35.

Hippius, H., Möller, H. -J., Müller, N., \& NeundöferKohl, G. (2008). The University Department of Psychiatric in Múnich: From Kraepelin and his predecessors to molecular psychiatry. Heidelberg: Springer.

Holdorff, B. (2004). Founding years of clinical neurology in Berlin until 1933. Journal of the History of the Neurosciences, 13(3), 223-238.

O Hospital Nacional de Alienados Comemora hoje 84 anos de sua fundação: O que é a Assistência Pública a Alienados no Distrito Federal, as homenagens que serão prestadas ao prof. Juliano Moreira. (18 de julio de 1925). Correio da Manhã, p. 05.

Humboldt, W., von (1997). Sobre a Organização Interna e Externa das Instituições Científicas Superiores em Berlim. En G. Casper \& W. von Humboldt (Eds.), Um mundo sem universidades? Rio de Janeiro: EdUERJ.

Jilek, W. G. (1995). Emil Kraepelin and comparative sociocultural psychiatry. European Archives of Psychiatry and Clinical Neuroscience, 245(4-5), 231-238.

Kraepelin, E. (1905). A paranoia. Arquivos Brasileiros de Psiquiatria, Neurologia e Ciências Afins, 1(1-4), 98-106.

Kraepelin, E. (1987). Memoirs. Berlin/Heidelberg: Springer-Verlag.

Kraepelin, E., Hippius, H., Peters, G., Ploog, D., Hoff, P., \& Kreuther, A. (Eds.). (1987). Emil Kraepelin. Memoirs. Berlin/Heidelberg: Springer-Verlag.

Magalhães, F. (1932). O centenário da Faculdade de Medicina do Rio de Janeiro, 1832-1932. Rio de Janeiro: TYP A. P. Barthel. 
Mauer, K., \& Mauer, U. (2006). Alzheimer: la vida de un médico y la historia de una enfermedad. Madrid: Ediciones Diaz de Santos.

Moreira, J. (1901a). A clínica psiquiátrica e de moléstias nervosas da Universidade de Leipzig. Revista do Grêmio dos Internos dos Hospitais, 2(1), 2-5.

Moreira, J. (1901b). A clínica psiquiátrica e de moléstias nervosas da Universidade de Leipzig. Revista do Grêmio dos Internos dos Hospitais, 2(2), 20-23.

Moreira, J. (1902). Da necessidade da fundação de laboratórios nos hospitais. Gazeta Médica da Bahia, 33(10), 439-450.

Moreira, J. (1905a). Briefe aus dem Jahr 1905: Juliano Moreira an Emil Kraepelin, Rio de Janeiro, 10. April. En W. Burgmair, E. Engstrom \& Weber, M. (Eds.), Emil Kraepelin in München (Vol. 1, pp. 213-214). Múnich: Belleville.

Moreira, J. (1905b). Briefe aus dem Jahr 1905: Juliano Moreira an Emil Kraepelin, Rio de Janeiro, 30. Juli. En W. Burgmair, E. Engstrom \& Weber, M. (Eds.), Emil Kraepelin in München (Vol. 1, pp. 228-229). Múnich: Belleville.

Moreira, J. (1909). Progress of Psychiatry in 1909: Brazil. The British Journal of Psychiatry, 56(234), 519-521.

Moreira, J. (1910). Quais os melhores meios de assistência aos alienados? Arquivos Brasileiros de Psiquiatria, Neurologia e Medicina Legal, 6(3-4), 373-396.

Moreira, J., \& Vianna, U. (1913). Die Allgemeine progressive Paralyse bei Greisen. Zeitschrift für die Gesamte Neurologie und Psychiatrie, 18(1), 187-194.

Moses, A. (1909). Do método biológico de diagnóstico em neurologia e psiquiatria. Arquivos Brasileiros de Psiquiatria, Neurologia e Medicina Legal, 5(1), 32-58.

Muñoz, P. F. N., \& Facchinetti, C. (2011). Diagnóstico y tratamiento en el Hospital Nacional de Alienados: el caso de los estados atípicos de degeneración a comienzos del siglo XX. Frenia. Revista de Historia de la Psiquiatría, 11, 89-107.

Muñoz, P. F. N., Facchinetti, C., \& Dias, A. A. T. (2011). Suspeitos em observação nas redes da psiquiatria: o Pavilhão de Observações (1894-1930). Memorandum, 20, 83-104.

Passos, A. (1975). Juliano Moreira (vida e obra). Rio de Janeiro: Livraria São José.

Pereira, M. E. C. (diciembre de 2007). Griesinger e as bases da "Primeira psiquiatria biológica". Revista
Latinoamericana de Psicopatologia Fundamental, 10(4). doi: 10.1590/S1415-47142007000400010

Piccinini, W. J., \& Bueno, J. R. (agosto de 2008). História da psiquiatria: Ulysses Viana Filho (1913-2000). Psychiatry on line Brasil, 13(7). Disponible em http://www.polbr.med.br/ano08/wal0808.php

Ribeiro, L. (1950). Afrânio Peixoto. Rio de Janeiro: Edições Conde.

Roxo, H. (1913). Doenças mentais e nervosas. BrasilMédico, 27(46), 503-505.

Roxo, H. (1921). Manual de psiquiatria. Rio de Janeiro: Editora Guanabara.

Shorter, E. (1997). A history of psychiatry: From the era of the asylum to the age of Prozac. New York: John Wiley \& Sons.

Silva, A. F. C. (2010). A trajetória de Henrique da Rocha Lima e as relações teuto-brasileiras (1901-1956). História, Ciências, Saúde-Manguinhos, 17, 495-509.

Sirinelli, J. -F. (2003). Os intelectuais. En R. Rémond (Org.), Por uma história política (pp. 271-294). Rio de Janeiro: FGV.

Smith, R. P. (1910). The international committee for the study of the causes and prophylaxis of mental disease. The British Journal of Psychiatry, 56(234), 389-405.

Souza, R. F. G. T. (2000). Mutações diagnósticas: a propósito da psicose unitária (Dissertação de Mestrado em Psiquiatria). Faculdad de Medicina, Universidade do Porto.

Spielmeyer, W. (1927). Kraepelin und die naturwissenschaftlich-medizinische Forschung in der Psychiatrie. Zeitschrift für die Gesamte Neurologie und Psychiatrie, 108(1), 10-20.

Teive, H. A. G., Almeida, S. M., De, \& Werneck, L. C. (2006). A contribuição brasileira para o estudo da neurocisticercose: o papel de Moses e Lange no diagnóstico liquórico. Arquivos de Neuro-Psiquiatria, 64(2b), 534-537.

Venâncio, A. T. A. (2003). Ciência psiquiátrica e política assistencial: a criação do Instituto de Psiquiatria da Universidade do Brasil. História, Ciências, Saúde Manguinhos, 10(3), 883-900.

Vianna, U. (1911). Contribuição ao diagnóstico da arterio-esclerose cerebral (estudo clínico e histopatológico) (Tesis de Medicina). Faculdade de Medicina, Rio de Janeiro. 
Vida Social: viajantes. (10 de marzo de 1912). O PAIZ, p. 05.

Weber, M. M. (1996). Ernst Rüdin, 1874-1952: A German psychiatrist and geneticist. American Journal of Medical Genetics, 67(4), 323-331.
Weber, M. M. (2000). Psychiatric research and science policy in Germany. The history of the Deutsche Forschungsanstalt für Psychiatrie (German Institute for Psychiatric Research) in Munich from 1917 to 1945. History of Psychiatry, 11(43), 235-258. 
